Schr odi nger equat i on i $n$ terms of I i near I y aver aged positi on moments

\begin{tabular}{|c|c|}
\hline 著者 & KOGA Toshi kat su \\
\hline $\begin{array}{l}\text { j our nal or } \\
\text { publ i cat i on } \mathrm{titl} \text { e }\end{array}$ & The j our nal of chemical physi cs \\
\hline vol une & 91 \\
\hline nunber & 8 \\
\hline page $r$ ange & 47744778 \\
\hline year & $1989-10-15$ \\
\hline URL & ht t p: //hdl . handl e. net /10258/939 \\
\hline
\end{tabular}




\title{
Schrodinger equation in terms of linearly averaged position moments
}

\author{
Toshikatsu Koga \\ Department of Applied Chemistry and Department of Applied Science for Energy, Muroran Institute of \\ Technology, Muroran, Hokkaido 050, Japan
}

(Received 26 June 1989; accepted 13 July 1989)

Linearly averaged position moment (LAPM) $\left[R_{1}^{k_{1}} R_{2}^{k_{2}} \cdots R_{3 N}^{k_{3 N}}\right]$ is defined as the linear (not quadratic) average of the position moment operator $R_{1}^{k_{1}} R_{2}^{k_{2}} \cdots R_{3 N}^{k_{3 N}}$ over the $N$-electron wave function $\Psi\left(\left\{R_{j}\right\}\right)$, where $\left\{R_{j}\right\}$ are $3 N$ Cartesian coordinates of electrons and $\left\{k_{j}\right\}$ are nonnegative integers. When all the LAPM's are well defined, it is shown that the Schrödinger equation is equivalent to a set of an infinite number of equations between LAPM's involving the potential-energy operator. The kinetic energy operator does not appear. The spherical polar representation of the LAPM equation is also presented. Illustrations are given for simple one- and two-electron atoms, where the LAPM equation is applied to the determination of approximate wave functions and associated energies.

\section{INTRODUCTION}

Previously, the zero momentum energy expression has been proposed ${ }^{1-3}$ and applied ${ }^{1-5}$ as a sensitive criterion to assess the accuracy of approximate wave functions. The expression has been derived by the application of the local energy concept ${ }^{6,7}$ to the momentum-space Schrödinger equation: The equation to be satisfied at the momentumspace origin is considered and is inversely transformed into position space. The resultant zero momentum energy expression includes rather strange integrals,

$$
\begin{aligned}
& {\left[\prod_{j=1}^{3 N} R_{j}^{k_{j}}\right] \equiv \int\left(\prod_{j=1}^{3 N} R_{j}^{k_{j}}\right) \Psi(\mathbf{r}) d \mathbf{r},} \\
& {\left[\left(\prod_{j=1}^{3 N} R_{j}^{k_{j}}\right) V(\mathbf{r})\right] \equiv \int\left(\prod_{j=1}^{3 N} R_{j}^{k_{j}}\right) V(\mathbf{r}) \Psi(\mathbf{r}) d \mathbf{r},}
\end{aligned}
$$

which are linear averages of the Cartesian moment $\mathrm{II}_{j=1}^{3 N} R_{j}^{k_{j}}$ and potential-energy $V(r)$ operators over the position wave function $\Psi(\mathbf{r})$ (see also Ref. 8 ). $\left\{k_{j}\right\}$ are non-negative integers.

Stimulated by these studies, we have recently shown ${ }^{9}$ that there exists a set of relations between position moments linearly averaged over the wave function. These relations constitute a necessary condition for the true wave function, and the zero momentum energy formula ${ }^{1-3}$ follows from the simplest case of the relations. A further generalization is proposed $^{10}$ for the linear average over the wave function in a finite domain, whose results can be used as a measure for the regional accuracy of wave functions. These studies have added nontrivial information to the structure of the Schrödinger equation.

In the present paper, we show that the set of relations between linearly averaged position moments (LAPM's) has a deterministic property. When all the LAPM's are well defined, the Schrödinger equation is equivalent to a set of an infinite number of equations between LAPM's involving the potential-energy operator $V(\mathbf{r})$. The kinetic energy operator becomes implicit. In the next section, the formalism is presented. Some illustrative applications are given in Sec. III for the determination of approximate wave functions and associated energies from the LAPM equation. Hartree atomic units are used throughout this paper.

\section{FORMALISM}

The $N$-electron Schrödinger equations in position and momentum spaces are written as

$$
\begin{aligned}
& (-\Delta / 2+V(\mathbf{r})-E) \Psi(\mathbf{r})=0, \\
& \left(p^{2} / 2-E\right) \Phi(\mathbf{p})+\int W\left(\mathbf{p}-\mathbf{p}^{\prime}\right) \Phi\left(\mathbf{p}^{\prime}\right) d \mathbf{p}^{\prime}=0,
\end{aligned}
$$

where $\mathbf{r} \equiv\left\{\mathbf{r}_{j}\right\}$ and $\mathbf{p} \equiv\left\{\mathbf{p}_{j}\right\}$ are position and momentum vectors of the electrons, respectively, and $\Delta \equiv \Sigma_{j=1}^{N} \Delta_{j}$ and $p^{2} \equiv \Sigma_{j=1}^{N}\left|\mathbf{p}_{j}\right|^{2}$. The wave functions $\Psi(\mathbf{r})$ and $\Phi(\mathbf{p})$ and the potential-energy operators $V(\mathbf{r})$ and $W(\mathbf{p})$ are, respectively, pairs of the Fourier transforms:

$$
\begin{aligned}
& \Phi(\mathbf{p})=(2 \pi)^{-3 N / 2} \int \exp (-i \mathbf{r} \cdot \mathbf{p}) \Psi(\mathbf{r}) d \mathbf{r}, \\
& \Psi(\mathbf{r})=(2 \pi)^{-3 N / 2} \int \exp (+i \mathbf{r} \cdot \mathbf{p}) \Phi(\mathbf{p}) d \mathbf{p}, \\
& W(\mathbf{p})=(2 \pi)^{-3 N} \int \exp (-i \mathbf{r} \cdot \mathbf{p}) V(\mathbf{r}) d \mathbf{r}, \\
& V(\mathbf{r})=\int \exp (+i \mathbf{r} \cdot \mathbf{p}) W(\mathbf{p}) d \mathbf{p},
\end{aligned}
$$

where $\mathbf{r} \cdot \mathbf{p}$ means $\sum_{j=1}^{N} \mathbf{r}_{j} \cdot \mathbf{p}_{j}$

Combining Eqs. (2)-(4), we obtain the Schrödinger equation in an intermediate representation:

$\left(p^{2} / 2-E\right)[\exp (-i \mathbf{r} \cdot \mathbf{p})]+[\exp (-i \mathbf{r} \cdot \mathbf{p}) V(\mathbf{r})]=0$,

where the square brackets stand for

$$
[f(\mathbf{r})] \equiv \int f(\mathbf{r}) \Psi(\mathbf{r}) d \mathbf{r}
$$

which is a linear average of $f(\mathbf{r})$ over the position wave function $\Psi(r)$. We implicitly assume that the two integrals appearing in Eq. (5) do exist. 


\section{A. Cartesian representation}

Now we expand the exponential function $\exp (-i \mathbf{r} \cdot \mathbf{p})$ in an absolutely convergent series:

$$
\begin{aligned}
\exp (-i \mathrm{r} \cdot \mathrm{p}) & =\prod_{j=1}^{3 N} \sum_{k_{j}=0}^{\infty}\left(-i R_{j} P_{j}\right)^{k_{1} / k_{j} !} \\
& =\sum_{\left\{k_{j}\right\}=0}^{\infty}(-i)^{\Sigma_{j} k_{j}}\left(R_{1} P_{1}\right)^{k_{1} \cdots\left(R_{3 N} P_{3 N}\right)^{k_{i N}} / k_{1} ! \cdots k_{3 N} !,}
\end{aligned}
$$

where $\left\{R_{j}\right\}$ and $\left\{P_{j}\right\}(j=1,2, \ldots, 3 N)$ represent the $3 N$ Cartesian coordinates of the position and momentum vectors of the $N$ electrons. We assume all the LAPM's $\left[I_{j=1}^{3 N} R_{j}^{k_{j}}\right]$ and $\left[\left(I_{j=1}^{3 N} R_{j}^{k_{j}}\right) V(\mathbf{r})\right]$ exist and their linear combinations are well defined. Then we can exchange the integration and the summation when Eq. (7) is substituted into Eq. (5), and we have

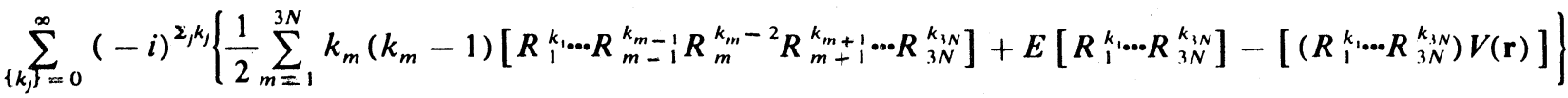

$$
\begin{aligned}
& \times\left(P_{1}^{k_{1} \ldots P_{3 N}} k_{3 N}^{k_{N}}\right) /\left(k_{1} ! \cdots k_{3 N} !\right)=0 \text {, }
\end{aligned}
$$

as an alternative form of Eq. (5). For Eq. (8) to hold for any values of $\left\{P_{j}\right\}$, all the coefficients of $P_{1}^{k_{1} \ldots .} P_{3 N}^{k_{3 N}}$ appearing on the left-hand side of Eq. (8) must vanish;

$$
\frac{1}{2} \sum_{m=1}^{3 N} k_{m}\left(k_{m}-1\right)\left[R_{m}^{-2} \prod_{j=1}^{3 N} R_{j}^{k_{j}}\right]+E\left[\prod_{j=1}^{3 N} R_{j}^{k_{j}}\right]-\left[\left(\prod_{j=1}^{3 N} R_{j}^{k_{j}}\right) V(\mathbf{r})\right]=0,
$$

where $k_{j}=0,1,2, \ldots$. Equation (9) represents a set of an infinite number of equations, and is equivalent to the Schrödinger equation provided that all the LAPM's and their linear combinations are well defined. It may be interesting to observe that in Eq. (9) the kinetic energy operator does not appear and its contribution is represented by the first term.

If we set $k_{2}=k_{3}=\cdots=k_{3 N}=0$ in Eq. (9), we obtain a special form:

$$
\frac{1}{2} k_{1}\left(k_{1}-1\right)\left[R_{1}^{k_{1}-2}\right]+E\left[R_{1}^{k_{1}}\right]-\left[R_{1}^{k_{i}} V(\mathbf{r})\right]=0,
$$

which is a set of single-component equations and constitutes a subset of Eq. (9).

\section{B. Spherical polar representation}

When the system under consideration has a spherical symmetry, the use of the spherical polar coordinates is natural. The relation to be satisfied between the LAPM's in the spherical polar coordinates can be derived as follows.

We first invoke the plane-wave expansion"

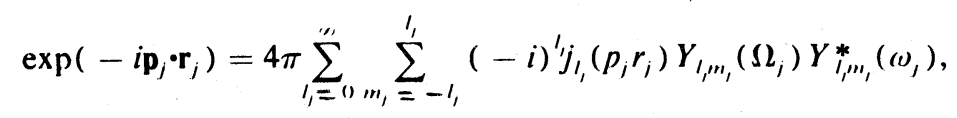

where $\left(p_{j}, \Omega_{j}\right)$ and $\left(r_{j},\left(\omega_{j}\right)\right.$ are the spherical polar coordinates of the vectors $\mathbf{p}_{j}$ and $\mathbf{r}_{j}$, respectively. $Y_{l m}$ is the usual spherical harmonic ${ }^{\prime \prime}$ and $j_{l}(z)$ is the spherical Bessel function of the first kind. ${ }^{2}$ Substituting Eq. (11) into the parent equation (5) and gathering the coefficients of the linearly independent term $\mathrm{II}_{i=1}^{N} Y_{l_{i, n_{l}}}\left(\Omega_{j}\right)$, we obtain

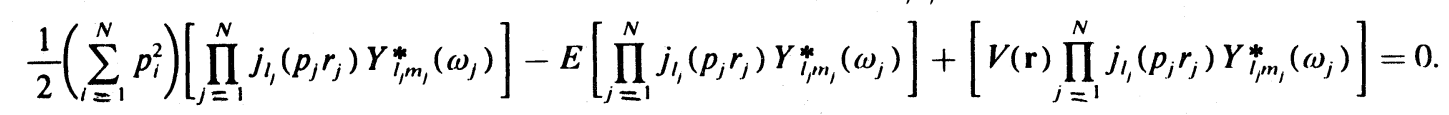

Substitution of the expansion ${ }^{12}$ for $j_{l}(z)$

$$
j_{l}(z)=(2 z)^{l} \sum_{k=0}^{\prime \prime \prime}\left\{(-1)^{k}(l+k) ! / k !(2 l+2 k+1) !\right\} z^{2 k}
$$

into Eq. (12) followed by comparison of the coefficients of $\mathrm{II}_{j}^{N}, p_{j}^{l_{j}+2 k_{\text {g }}}$ gives the desired result:

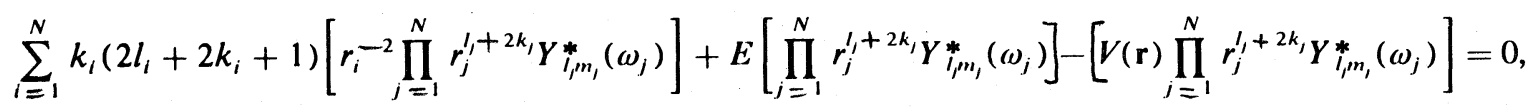

where $k_{i}=0,1,2, \ldots ; l_{i}=0,1,2, \ldots ; m_{i}=-l_{i},-l_{i}+1, \ldots,+l_{i}$ for $i=1,2, \ldots, N$. Equation (14) is a set of LAPM equations in the spherical polar coordinates.

A special case of Eq. (14) for $l_{i}=m_{i}=0(i=1,2, \ldots, N)$ reads

$$
\sum_{i=1}^{N} k_{i}\left(2 k_{i}+1\right)\left[r_{i}^{-2} \prod_{j=1}^{N} r_{j}^{2 k_{j}}\right]+E\left[\prod_{j=1}^{N} r_{j}^{2 k_{j}}\right]-\left[\left(\prod_{j=1}^{N} r_{j}^{2 k_{i}}\right) V(\mathbf{r})\right]=0
$$

since $Y_{(0)}\left(\omega_{j}\right)=(4 \pi)^{-1 / 2}$. If we set $k_{2}=k_{3}=\cdots=k_{N}=0$, Eq. (15) further reduces to a set of single-component equations

$$
k_{1}\left(2 k_{1}+1\right)\left[r_{1}^{2 k_{1}-2}\right]+E\left[r_{1}^{2 k_{1}}\right]-\left[r_{1}^{2 k_{1}} V(\mathbf{r})\right]=0 \text {, }
$$

which may compare with Eq. (10) in the Cartesian representation. 


\section{Discussion}

Equation (9) may be considered as a projection of the position-space Schrödinger equation onto momentum space with the discrete Cartesian bases $\mathrm{II}_{j=1}^{3 N} P_{j}^{k_{j}}$ defined by a set of non-negative integers $\left\{k_{j}\right\}$. Similarly, Eq. (14) is regarded as a projection onto the spherical polar bases $\mathrm{II}_{j=1}^{N} p_{j}^{l_{j}+2 k_{j}} Y_{l_{j} m_{j}}\left(\Omega_{j}\right)$ defined by the integers $\left\{k_{j}, l_{j}, m_{j}\right\}$. We can then use Eq. (9) or (14) for the determination of an approximate wave function and the associated energy. When a trial function includes $M$ parameters, $M+1$ equations from the set (9) or (14) [or the subset (10), (15), or (16)] determine the values of these parameters as well as the approximate energy $E$. In this approach, no existing approximation theories are invoked such as the variation method and the perturbation theory.

Then a problem of how to choose the $M+1$ equations from the set (9) or (14) emerges. In the case of the Cartesian representation, we should be reminded that an equation from Eq. (9) with some $\left\{k_{j}\right\}$ constitutes the coefficient of the bases $I_{j=1}^{3 N} P_{j}^{k_{j}}$ when the Schrödinger equation (5) is expanded. If we adopt equations with smaller (larger) $\left\{k_{j}\right\}$, the Schrödinger equation is satisfied in a smaller (larger) $\mathbf{p}$ region of momentum space. Therefore the choice depends on which region one needs more accurate description for. Here we note that the position and momentum representations emphasize inverse regions of the respective spaces. Analogous discussion holds for the set (14) given in the spherical polar coordinates.

\section{ILLUSTRATIVE APPLICATIONS}

In the following, we apply the LAPM equation to (approximate) solutions of two simple systems, i.e., the hydrogen-like atom and the ground-state helium atom. In the former case, the correct wave function and the associated eigenenergy are completely reproduced. In the latter case, we discuss the property of approximate LAPM solutions using a simple trial function.

\section{A. Hydrogen-like atom}

For a single-electron system, the LAPM equation in the spherical polar coordinates [Eq. (14)] reads

$$
k(2 k+2 L+1)\left[r^{2 k+L-2} Y_{L M}^{*}(\omega)\right]+E\left[r^{2 k+L} Y_{L M}^{*}(\omega)\right]-\left[V(\mathbf{r}) r^{2 k+L} Y_{L M}^{*}(\omega)\right]=0 .
$$

When $V(\mathbf{r})=-Z / r$ (i.e., a hydrogen-like atom with nuclear charge $Z$ ), Eq. (17) suggests that the wave function $\Psi(\mathbf{r})$ has the following form:

$$
\Psi(\mathbf{r})=R(r) Y_{l m}(\omega) .
$$

Substituting Eq. (18) and the explicit form of $V(\mathbf{r})$ into Eq. (17), we obtain the radial LAPM equation:

$$
k(2 k+2 l+1)\left[r^{2 k+l-2}\right]_{r}+E\left[r^{2 k+l}\right]_{r}+Z\left[r^{2 k+l-1}\right]_{r}=0
$$

where

$$
[f(r)]_{r} \equiv \int_{0}^{\infty} f(r) R(r) r^{2} d r .
$$

If we assume a radial function $R(r)$ of the form

$$
R(r)=\left(\sum_{i=0}^{n-1} a_{i} \zeta^{i} r^{i}\right) \exp (-\zeta r),
$$

Eq. (19) becomes

$$
k(2 k+2 l+1) \sum_{i=0}^{n-1} a_{i}(2 k+l+i) !+\left(E / \zeta^{2}\right) \sum_{i=0}^{n-1} a_{i}(2 k+l+i+2) !+(Z / \zeta) \sum_{i=0}^{n-1} a_{i}(2 k+l+i+1) !=0,
$$

from which we have to determine $E,\left\{a_{i}\right\}$, and $\zeta$ for a given set of $Z, l$, and $n$.

To solve Eq. (22), we introduce a linearly independent basis set defined by

$$
(x)_{i} \equiv \begin{cases}(x+1)(x+2) \cdots(x+i) & \text { for } i \geqslant 1, \\ 1 & \text { for } i=0 .\end{cases}
$$

Then Eq. (22) is rearranged as

$$
\begin{aligned}
\left(\frac{1}{2}+\right. & \left.E / \zeta^{2}\right) a_{n-1}(2 k+l)_{n+1}+\left\{\left(\frac{1}{2}+E / \zeta^{2}\right) a_{n-2}+(Z / \zeta-n) a_{n-1}\right\}(2 k+l)_{n}+\sum_{i=2}^{n-1}\left\{\left(\frac{1}{2}+E / \zeta^{2}\right) a_{i-2}\right. \\
& \left.+(Z / \zeta-i) a_{i-1}-\frac{1}{2}(l-i)(l+i+1) a_{i}\right\}(2 k+l)_{i} \\
& +\left\{(Z / \zeta-1) a_{0}-\frac{1}{2}(l-1)(l+2) a_{1}\right\}(2 k+l)_{1}-\frac{1}{2} l(l+1) a_{0}(2 k+l)_{0}=0
\end{aligned}
$$

For Eq. (24) to hold for any non-negative integer $k$, all the coefficients of $\left\{(2 k+l)_{i}\right\}$ must vanish. We immediately have

$$
\zeta=Z / n, \quad E=-\zeta^{2} / 2=-Z^{2} / 2 n^{2},
$$


and a recursion relation for the expansion coefficients $\left\{a_{i}\right\}$

$$
\begin{aligned}
& (l-i)(l+i+1) a_{i}=2(n-i) a_{i-1}, \quad i=1,2, \ldots, n-1, \\
& l(l+1) a_{0}=0 .
\end{aligned}
$$

When $n<l, l-i$ is positive and therefore Eq. (26a) has a general solution

$$
a_{i}=\left\{2^{i} l(l+1)(n-1) !(l-i-1) ![(n-i-1) !(l+i+1) !]^{-1}\right\} a_{0} .
$$

Because of Eq. (26b), however, Eq. (27a) trivially results in

$$
a_{i}=0, \quad i=0,1, \ldots, n-1,
$$

and there is no physically meaningful solution for the case of $n<l$. The well-known quantum condition $n>l$ or $l<n-1$ thus emerges. In this case, Eqs. (26a) and (26b) have a general solution

$$
a_{i}= \begin{cases}\left\{(-2)^{1-l}(n-l-1) !(2 l+1) ! /(n-i-1) !(i-l) !(i+l+1) !\right\} a_{l} & \text { for } l<i<n-1, \\ 0 & \text { for } 0 \leqslant i<l,\end{cases}
$$

where $a_{l}$ is an arbitrary nonzero constant.

Now the radial function $R(r)$ [Eq. (21)] becomes

$$
\begin{aligned}
R(r) & =\left(\sum_{i=1}^{n-1} a_{i} \zeta^{i} r^{i}\right) \exp (-\zeta r) \\
& =(\zeta r)^{\prime}\left(\sum_{i=0}^{n-1-1} a_{i+1} \zeta^{i} r^{i}\right) \exp (-\zeta r),
\end{aligned}
$$

where

$$
\begin{aligned}
a_{l+l}= & \left\{(-2)^{\prime}(n-l-1) !(2 l+1) !\right. \\
& \left.\times[(n-l-i-1) !(2 l+i+1) ! i !]^{-1}\right\} a_{l},
\end{aligned}
$$

and $\zeta=Z / n$. If we choose

$$
a_{l}=-[(n+l-1) !]^{2} /[(n-l-1) !(2 l+1) !]
$$

in Eq. (29b), the polynomial involved in Eq. (29a) is nothing but the associated Laguerre polynomial $L_{n+i}^{2 l+1}(2 \zeta r)$. We finally have

$$
R(r)=(\zeta r)^{\prime} L_{n+1}^{2 l+1}(2 \zeta r) \exp (-\zeta r) .
$$

Equations (18), (25), and (30) constitute the complete solutions for the hydrogen-like atom obtained from the present LAPM equation. They are identical to the known results ${ }^{1.3}$ which usually follow from the solution of the Schrödinger equation as a second-order differential equation.

\section{B. Ground-state helium atom}

The LAPM equation (15) for $N=2$ is

$$
\begin{gathered}
k_{1}\left(2 k_{1}+1\right)\left[r_{1}^{2 k_{1}-2} r_{2}^{2 k_{2}}\right]+k_{2}\left(2 k_{2}+1\right)\left[r_{1}^{2 k_{1}} r_{2}^{2 k_{2}-2}\right] \\
+E\left[r_{1}^{2 k_{1}} r_{2}^{2 k_{2}}\right]-\left[r_{1}^{2 k_{1}} r_{2}^{2 k_{2}} V\left(\mathbf{r}_{1}, \mathbf{r}_{2}\right)\right]=0,
\end{gathered}
$$

where

$$
V\left(\mathbf{r}_{1}, \mathbf{r}_{2}\right)=-Z / r_{1}-Z / r_{2}+1 / r_{12},
$$

for a helium-like atom with nuclear charge $Z$. Let us consider the approximate wave function given by a single product of the scaled hydrogenic $1 s$ functions:

$$
\Psi\left(\mathbf{r}_{1}, \mathbf{r}_{2}\right)=\exp \left(-\zeta r_{1}\right) \exp \left(-\zeta r_{2}\right),
$$

which was first examined by Kellner't in the variational treatment of the ground-state helium atom.

For a special case of $k_{1}=k$ and $k_{2}=0$, the explicit evaluation of the integrals appearing in Eq. (31) for the wave function (33) results in a simplified LAPM equation

$$
\begin{aligned}
k \zeta^{2} & +2(k+1) E \\
& +\left\{(k+2) Z-1+(k+3) 2^{-2 k-3}\right\} \zeta=0,
\end{aligned}
$$

which includes the quantities $E$ and $\zeta$ to be determined. In contrast to the case of hydrogen-like atoms, there is no set of $E$ and $\zeta$ values which satisfies Eq. (34) for all possible $k$, implying that the exact wave function is not expressed in the form of Eq. (33).

However, we can employ some selected equations from Eq. (34) for an approximate determination of $E$ and $\zeta$. Let us examine the first two equations resulting from $k=0$ and 1. They are

$$
\begin{aligned}
& E+(Z-5 / 16) \zeta=0, \quad \text { for } k=0, \\
& \zeta^{2}+4 E+(3 Z-7) \zeta=0, \quad \text { for } k=1 .
\end{aligned}
$$

Though we cannot uniquely determine two unknowns from a single equation, Eq. (35a) holds if

$\zeta=\zeta_{0}=Z-{ }_{10}^{5}$ and $E=E_{0}=-\zeta_{0}^{2}=-\left(Z-\gamma_{0}^{5}\right)^{2}$,

which are $\zeta_{0}=1.6875$ and $E_{0}=-2.847656$ when $Z=2$. Accidentally, Eq. (36a) is identical to the result of the variational calculation as has been discussed in relation to the zero momentum energy expression.' Similarly, Eq. (35b) holds if

$\zeta=\zeta_{1}=Z-{ }_{24}^{7}$ and $E=E_{1}=-\zeta_{1}^{2}=-\left(Z-{ }_{24}^{7}\right)^{2}$,

which become $\zeta_{1}=1.708333$ and $E_{1}=-2.918403$ when $Z=2$. This approximate energy $E_{1}$ is lower than the exact energy $^{15}-2.903724$, since the LAPM energy is not an upper bound to the true energy in general. The unique solutions that satisfy both Eqs. (35a) and (35b) are

$$
\begin{aligned}
& \zeta=\zeta_{01}=Z-\frac{3}{8} \text { and } \\
& E=E_{01}=-\left(Z-5_{6}^{5}\right)\left(Z-\frac{3}{8}\right),
\end{aligned}
$$

which become $\zeta_{01}=1.625$ and $E_{01}=-2.742188$ for $Z=2$.

In order to clarify the difference in the above three approximate solutions [ Eqs. (36a), (36b), and (36c)], it may be convenient to introduce the quantity

$$
\begin{aligned}
\Delta\left(\mathbf{p}_{1}, \mathbf{p}_{2}\right) \equiv & \left.\left(p_{1}^{2} / 2+p_{2}^{2} / 2-E\right) \mid \exp \left(-i \mathbf{r}_{1} \cdot \mathbf{p}_{1}-i \mathbf{r}_{2} \cdot \mathbf{p}_{2}\right)\right] \\
& +\left[\exp \left(-i \mathbf{r}_{1} \cdot \mathbf{p}_{1}-i \mathbf{r}_{2} \cdot \mathbf{p}_{2}\right) V\left(\mathbf{r}_{1}, \mathbf{r}_{2}\right)\right] .
\end{aligned}
$$


The condition $\Delta\left(\mathbf{p}_{1}, \mathbf{p}_{2}\right)=0$ is equivalent to the Schrödinger equation in momentum space, and hence the deviation of $\Delta\left(\mathbf{p}_{1}, \mathbf{p}_{2}\right)$ from zero characterizes the property of an approximate solution. For a particular case of $\mathbf{p}_{2}=\mathbf{0}$, Eq. (37) reduces to

$$
\begin{aligned}
\delta\left(\mathbf{p}_{1}\right) \equiv & \Delta\left(\mathbf{p}_{1}, \mathbf{0}\right) \\
= & \left(p_{1}^{2} / 2-E\right)\left[\exp \left(-i \mathbf{r}_{1} \cdot \mathbf{p}_{1}\right)\right] \\
& +\left[\exp \left(-i \mathbf{r}_{1} \cdot \mathbf{p}_{1}\right) V\left(\mathbf{r}_{1}, \mathbf{r}_{2}\right)\right] .
\end{aligned}
$$

For the approximate wave function given by $\mathrm{Eq}$. (33), the difference function $\delta(\mathbf{p})$ is evaluated to be

$$
\begin{aligned}
\delta(\mathbf{p})= & \delta(p)=32 \pi^{2}\left\{\left(p^{2}-2 E\right) \zeta^{-2}\left(p^{2}+\zeta^{2}\right)^{-2}\right. \\
& -Z \zeta^{-1}\left(p^{2}+\zeta^{2}\right)^{-1}\left[\zeta^{-2}+\left(p^{2}+\zeta^{2}\right)^{-1}\right] \\
& +2 \zeta^{2}\left[L_{6}(p ; \zeta, E)+L_{7}(p ; \zeta, E)\right. \\
& \left.\left.+L_{8}(p ; \zeta, E)\right]\right\}
\end{aligned}
$$

where the explicit forms of the functions $L_{j}(p ; \zeta, E)$ $(j=6,7,8)$ are given in Ref. 16.

In the case of the helium atom $(Z=2)$, Fig. 1 shows the $\delta(p)$ functions for the three sets of approximate parameters, $\left(\zeta_{0}, E_{0}\right),\left(\zeta_{1}, E_{1}\right)$, and $\left(\zeta_{01}, E_{01}\right)$. For the first set obtained from the LAPM equation (34) with $k=0$, the deviation of $\delta(p)$ from zero is small in the low-momentum region and especially $\delta(0)=0$. For the second set resulting from the LAPM equation with $k=1,|\delta(p)|$ decreases in the highmomentum region, but increases remarkably in the low-momentum region. For the third set, the low-momentum behavior of $\delta(p)$ is much improved, but a large deviation in the high-momentum region is observed. These behaviors may be easily understood if we are reminded of the fact that the satisfaction of the $k$ th LAPM equation corresponds to making the coefficient of $p^{2 k}$ zero when $\delta(p)$ is expanded in a power series of $p^{2}$. In fact, the $\delta(p)$ functions for the three sets of parameters can be expressed as

$$
\begin{aligned}
\delta_{0}(p)= & 32 \pi^{2}\left[\left(2^{24} / 3^{21}\right) p^{2}\right. \\
& \left.-\left(37 \cdot 2^{29} / 3^{27}\right) p^{4}+O\left(p^{6}\right)\right], \\
\delta_{1}(p)= & 32 \pi^{2}\left[\left(163 \cdot 2^{13} \cdot 3^{4} / 41^{5}\right)\right. \\
& \left.-\left(79 \cdot 2^{20} \cdot 3^{8} / 41^{9}\right) p^{4}+O\left(p^{6}\right)\right], \\
\delta_{01}(p)= & 32 \pi^{2}\left[-\left(21 \cdot 2^{20} / 13^{9}\right) p^{4}+O\left(p^{6}\right)\right] .
\end{aligned}
$$

The low-momentum superiority of the third set [Eq. (36c)] is clear from Eq. (40c). If we adopt a more flexible trial function with several adjustable parameters, we may be able to extend the zero deviation of the $\delta(p)$ function to the intermediate-momentum region. Such a possibility will be examined in the future.

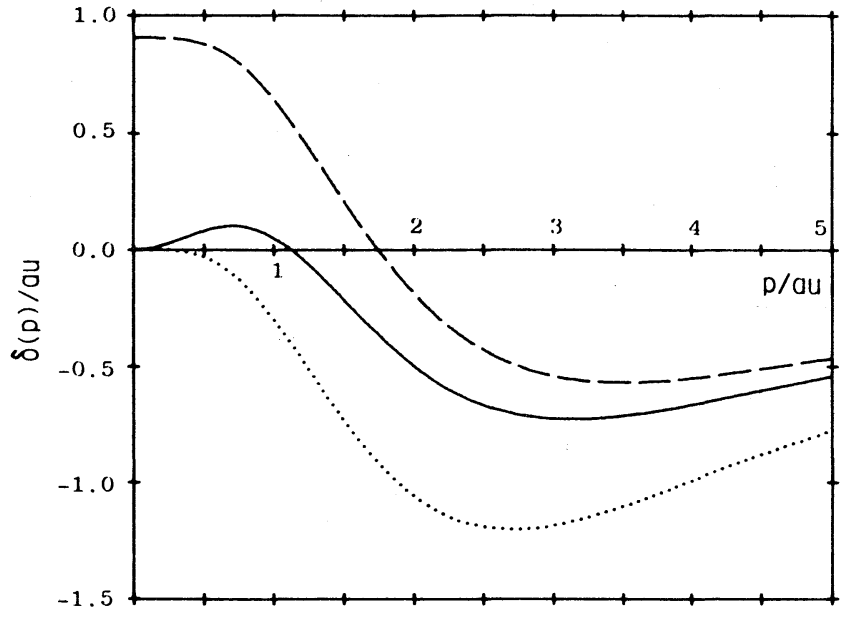

FIG. 1. Behavior of the difference function $\delta(p)$ for the three sets of parameters $\zeta$ and $E$ with $Z=2$. The solid line is for $\zeta_{0}$ and $E_{0}$ [Eq. (36a)], the dashed line for $\zeta_{1}$ and $E_{1}$ [Eq. (36b)], and the dotted line for $\zeta_{01}$ and $E_{01}$ [Eq. (36c)].

\section{ACKNOWLEDGMENTS}

The author thanks Professor Hidenori Katsurada for helpful discussion on the solution of the LAPM equation for hydrogen-like atoms. Part of this study has been supported by a Grant-in-Aid for Scientific Research from the Ministry of Education of Japan.

'B. H. Armstrong, Bull. Am. Phys. Soc. 9, 401 ( 1964); Lockheed Missiles and Space Company Technical Report, Physics, No. 6-74-64-26, 1964.

${ }^{2}$ A. J. Thakkar and V. H. Smith, Jr., Phys. Rev. A 18, 841 (1978).

'T. Koga, K. Ohta, and A. J. Thakkar, Phys. Rev. A 37, 1411 (1988).

${ }^{4}$ R. J. Drachman, Phys. Rev. 136, A 641 (1964).

'T. Koga, J. Chem. Phys. 83, 6301 (1985).

'J. H. Bartlett, Phys. Rev. 51, 661 (1937).

${ }^{7}$ A. A. Frost, J. Chem. Phys. 10, 240 ( 1942); A. A. Frost, R. E. Kellog, and

E. C. Curtiss, Rev. Mod. Phys. 32, 313 (1960).

${ }^{\mathrm{x}}$ T. Koga and A. J. Thakkar, Int. J. Quantum Chem. 34, 103 (1988)

'T. Koga, J. Chem. Phys. 89, 5961 (1988)

I(1)T. Koga, J. Chem. Phys. 90, 5887 (1989)

"A. Messiah, Quantum Mechanics (North-Holland, Amsterdam, 1961), Vol. 1, pp. 494-497.

'"M. Abramowitz and I. A. Stegun, Handbook of Mathematical Functions (Dover, New York, 1972), p. $435 \mathrm{ff}$.

' 'See, for example, L. Pauling and E. B. Wilson, Introduction to Quantum Mechanics (McGraw-Hill, New York, 1935), Chap. 5.

'4G. W. Kellner, Z. Phys. 44, 91 (1927).

${ }^{15}$ K. Frankowski and C. L. Pekeris, Phys. Rev. 146, 46 (1966).

'P. J. Schreiber, R. P. Hurst, and T. E. Duvall, Phys. Rev. A 38, 3200 (1988). 